\title{
Controlling the spin-torque efficiency with ferroelectric barriers
}

\author{
A. Useinov, ${ }^{1}$ M. Chshiev, ${ }^{2,3,4}$ and A. Manchon ${ }^{1, *}$ \\ ${ }^{1}$ King Abdullah University of Science and Technology (KAUST), Physical Science and \\ Engineering Division, Thuwal 23955-6900, Saudi Arabia \\ ${ }^{2}$ Univ. Grenoble Alpes, INAC-SPINTEC, F-38000 Grenoble, France \\ ${ }^{3}$ CEA, INAC-SPINTEC, F-38000 Grenoble, France \\ ${ }^{4}$ CNRS, SPINTEC, F-38000 Grenoble, France
}

(Received 18 November 2012; revised manuscript received 10 December 2014; published 11 February 2015)

\begin{abstract}
Nonequilibrium spin-dependent transport in magnetic tunnel junctions comprising a ferroelectric barrier is theoretically investigated. The exact solutions of the free electron Schrödinger equation for electron tunneling in the presence of interfacial screening are obtained by combining Bessel and Airy functions. We demonstrate that the spin transfer torque efficiency, and more generally the bias dependence of tunneling magneto- and electroresistance, can be controlled by switching the ferroelectric polarization of the barrier. In particular, the critical voltage at which the in-plane torque changes sign can be strongly enhanced or reduced depending on the direction of the ferroelectric polarization of the barrier. This effect provides a supplementary way to electrically control the current-driven dynamic states of the magnetization and related magnetic noise in spin transfer devices.
\end{abstract}

DOI: 10.1103/PhysRevB.91.064412

PACS number(s): 72.25.-b, 77.55.Nv, 85.75.-d

The electrical control of magnetization in thin films is currently attracting intensive efforts due to its major potential for applications [1]. Current- and voltage-induced magnetization dynamics have been observed in a wide variety of magnetic heterostructures such as metallic and semiconducting spin valves and domain walls [2-5]. Of most technological interest is the manipulation of magnetization in magnetic tunnel junctions (MTJs) — trilayers comprising a tunnel barrier embedded between two ferromagnets-by means of either gate voltages or spin-polarized currents. In the first case, a large voltage pulse is applied across the barrier and charge reordering at the interface modifies the interfacial magnetic anisotropy [5]. In the second case, a spin-polarized current tunnels through the barrier and transfers its spin angular momentum to the local magnetization of the free magnetic layer [2-4]. This last phenomenon, known as spin transfer torque [6], enables the design of promising components such as on-chip tunable microwave generators [7], magnetic memory cells [8], race-track memories [9], and so on.

The most successful candidates to date that combine low critical switching current densities with large tunneling magnetoresistance ratios (TMR) are MTJs based on $\mathrm{MgO}$ barriers and transition metal electrodes [10] (i.e., Fe, Co, $\mathrm{Ni}$, and their compounds). Significant progress has been achieved towards understanding the complex microscopic nature of the junction's interfaces [11,12] and establishing the characteristics of spin transfer torque $[13,14]$. Despite undeniable successes $[15,16]$, the actual exploitation of spin transfer torque in devices is facing major hurdles, among which the necessary reduction of its critical switching current as well as the control of current-driven magnetic instabilities. Serious efforts are being made towards improving the device performances, and solutions such as engineering the junction structural asymmetries [17] or designing the metallic electrodes stacking [18] have been proposed to enhance the device

\footnotetext{
*aurelien.manchon@kaust.edu.sa
}

operation through modifying the bias dependence of the spin torque. However, little has been done on the barrier material itself and $\mathrm{MgO}$ is still considered as the best candidate for spin torque purposes.

Nonetheless, metal-oxides barriers display a wide variety of functional properties among which ferroelectricity can be used to add a degree of freedom to the system [19]. In oxide perovskytes, such as $\mathrm{BaTiO}_{3}$ or $\mathrm{BiFeO}_{3}$, uncompensated charge screening in the material induces a ferroelectric polarization which produces in turn very narrow charge depletion regions at the interfaces, as illustrated in Fig. 1. When inserted between nonmagnetic metallic electrodes, ferroelectric barriers have been theoretically predicted to result in large tunneling electroresistance effects (TER) [20-22], as recently reported experimentally [23-29]. The combination of ferroelectricity and ferromagnetism in a same material, referred to as a multiferroic, allows for the electric manipulation of the magnetization and vice versa [19]. However, the difficulty of growing multiferroic thin films limits their exploitation as viable memory elements for now. A promising route is to use synthetic multiferroics, where a ferroelectric barrier is inserted between two ferromagnets. In this case, the system displays gigantic TER ratios [30,31] resulting from the impact of the ferroelectric polarization on interfacial spin polarization [21,32,33]. Important progress has been realized in the interfacial control of magnetic properties [34-36], and ferroelectric junctions have been implemented in solid-state memory devices [37].

Whereas the aforementioned results are obtained using thick ferroelectric barriers $(>2 \mathrm{~nm})$, reducing the barrier thickness would enable spin transfer to occur. While the barrier reduction remains a challenging issue due to the emergence of pinholes and the formation of ferroelectric domains, the current advances are encouraging. In the present paper, we theoretically address the nature of the spin transfer torque in magnetic tunnel junctions comprising such a thin ferroelectric barrier. We demonstrate that the bias dependence of the spin transfer torque and magnetoresistance can be controlled by 
(a)
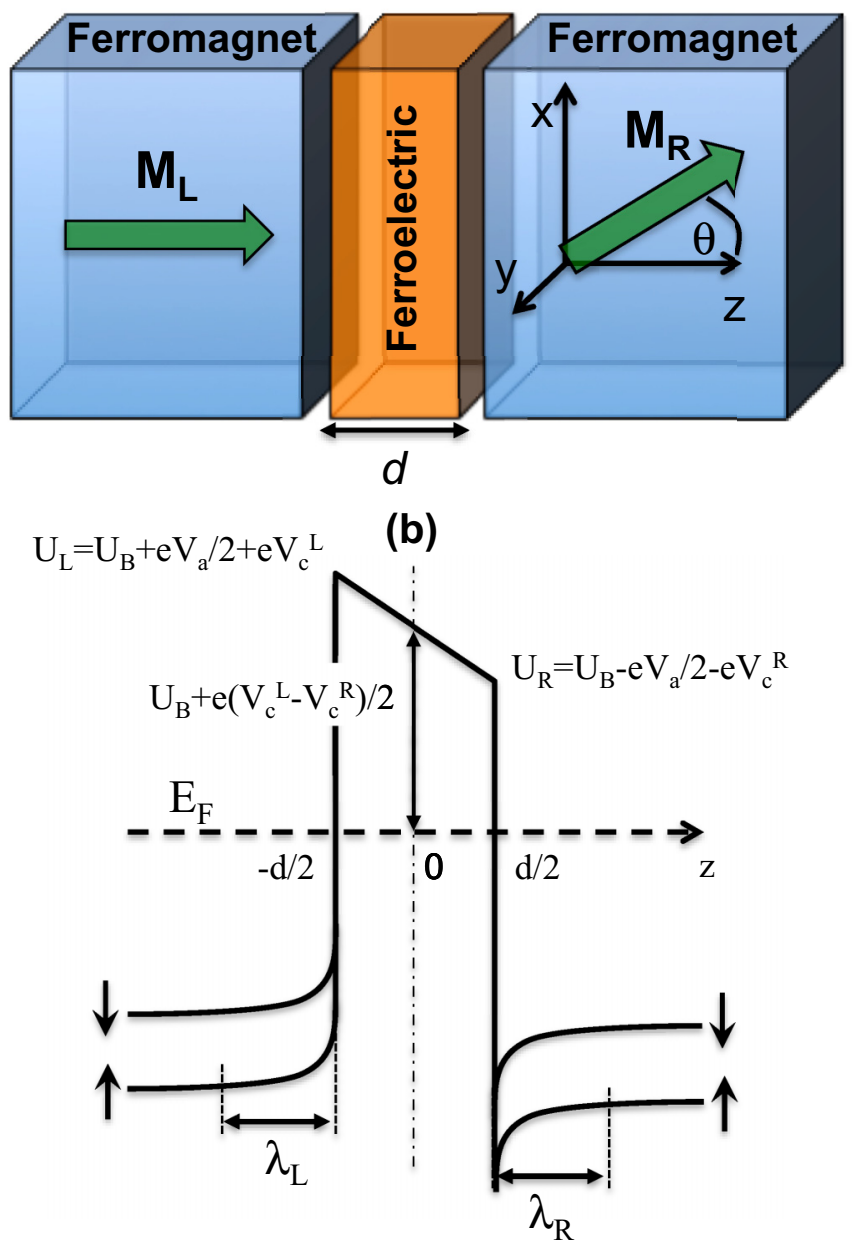

FIG. 1. (Color online) (a) Schematics of a magnetic tunnel junction comprising two ferromagnets and a ferroelectric insulator; (b) Potential profile of the junction with positive polarization (screening) and applied voltage $V_{a}$.

tuning the ferroelectric polarization, opening avenues for spin-based functional devices.

Let us consider a magnetic tunnel junction composed of $\mathrm{FM}_{\mathrm{L}} / \mathrm{FEI} / \mathrm{FM}_{\mathrm{R}}$, where $\mathrm{FM}_{\mathrm{L}(\mathrm{R})}$ is the semi-infinite left (right) ferromagnetic electrode and FEI represents a nonmagnetic ferroelectric insulator, such as $\mathrm{BaTiO}_{3}, \mathrm{PbTiO}_{3}$, $\mathrm{Pb}\left(\mathrm{Zr}_{0.2} \mathrm{Ti}_{0.8}\right) \mathrm{O}_{3}$, etc. The unit magnetization vectors are $\mathbf{M}_{\mathrm{L}}=\mathbf{e}_{z}$ and $\mathbf{M}_{\mathrm{R}}=\mathbf{e}_{x} \sin \theta+\mathbf{e}_{z} \cos \theta, \theta$ being the angle between the magnetization directions [Fig. 1(a)]. The junction is treated within a one-dimensional free-electron two-band Stoner model. The virtue of the free electron approach is to reduce the complexity of the band structure to a minimal set of parameters relevant to the spin transport, providing a qualitative description of the physics at stake. This method successfully predicted the gigantic TER effect by Zhuravlev et al. $[21,38]$ as well as the bias dependence of the spin torque in magnetic tunnel junctions $[13,14,17]$. Strictly speaking, this formalism applies in low band filling systems where the tunneling is dominated by electrons close to the $\Gamma$ point. It completely overlooks the Bloch nature of the tunneling electron states which might turn out to be important in single crystal samples and can be accounted for properly through $a b$ initio methods $[33,39]$. The Hamiltonian of the full system reads $\hat{H}=\hat{H}_{\mathrm{L}}+\hat{H}_{\mathrm{B}}+\hat{H}_{\mathrm{R}}$, where $[21,40]$

$$
\begin{gathered}
\hat{H}_{\mathrm{B}}=\frac{\hat{\mathbf{p}}^{2}}{2 m_{\mathrm{B}}}+U_{\mathrm{B}}+\frac{e}{2}\left(V_{\mathrm{c}}^{\mathrm{L}}-V_{\mathrm{c}}^{\mathrm{R}}\right)-\frac{z e}{d}\left(V_{a}+V_{\mathrm{c}}^{\mathrm{L}}+V_{\mathrm{c}}^{\mathrm{R}}\right), \\
\hat{H}_{\mathrm{L}(\mathrm{R})}=\frac{\hat{\mathbf{p}}^{2}}{2 m_{\mathrm{e}}}-\frac{\Delta_{\mathrm{L}(\mathrm{R})}(z)}{2} \hat{\boldsymbol{\sigma}} \cdot \mathbf{M}_{\mathrm{L}(\mathrm{R})} \pm \frac{e V(z)}{2},
\end{gathered}
$$

where

$$
\begin{gathered}
\Delta_{\mathrm{L}(\mathrm{R})}(z)=\Delta \mp 2 e V_{c}^{\mathrm{L}(\mathrm{R})} \frac{J \Delta \rho_{\mathrm{L}(\mathrm{R})}}{1+J \rho_{\mathrm{L}(\mathrm{R})}} e^{-(z \pm d / 2) / \lambda_{\mathrm{L}(\mathrm{R})}}, \\
e V(z)=e V_{a}+2 e V_{c}^{\mathrm{L}(\mathrm{R})} e^{-(z \pm d / 2) / \lambda_{\mathrm{L}(\mathrm{R})}},
\end{gathered}
$$

where $V_{\mathrm{c}}^{\mathrm{L}(\mathrm{R})}=\frac{d P \lambda_{\mathrm{L}(\mathrm{R})}}{\epsilon_{0} d+\epsilon_{\mathrm{f}}\left(\lambda_{\mathrm{L}}+\lambda_{\mathrm{R}}\right)}$ is the additional barrier potential due to the ferroelectric polarization at the left $(\mathrm{L})$ and right (R) interfaces, respectively, $\lambda_{i}=\frac{e^{2}}{\epsilon_{0}} \frac{\rho_{i}+4 J_{i} \rho_{i}^{\downarrow} \rho_{i}^{\uparrow}}{1+J_{i} \rho_{i}}$ is the screening length of the $i$ th electrode [40], and $d$ is the thickness of the barrier. $\rho^{\sigma}$ is the spin-dependent density of states with $\rho_{i}=\rho_{i}^{\uparrow}+\rho_{i}^{\downarrow}$ and $\Delta \rho_{i}=\rho_{i}^{\uparrow}-\rho_{i}^{\downarrow}, d$ is the thickness of the ferroelectric barrier, $\hat{\sigma}=\left(\hat{\sigma}_{x}, \hat{\sigma}_{y}, \hat{\sigma}_{z}\right)$ is the vector of the Pauli matrices, $\epsilon_{0}, \epsilon_{\mathrm{f}}$ are the dielectric static permittivity of the vacuum and ferroelectric barrier, respectively. $P$ represents the ferroelectric polarization, $\Delta=J \mathrm{~m}_{\mathrm{sd}}$ denotes the exchange energy, $\mathrm{m}_{\mathrm{sd}}=\left(\mathrm{n}^{\uparrow}-\mathrm{n}^{\downarrow}\right)$ is the equilibrium spin density, $\mathrm{n}^{\uparrow, \downarrow}=$ $\frac{1}{6 \pi^{2}}\left[\frac{2 m_{\mathrm{e}}}{\hbar^{2}}\left(\mathrm{E}_{\mathrm{F}} \pm \Delta / 2\right)\right]^{3 / 2}$ is the spin-dependent particle density, $J$ is Stoner exchange parameter, $e$ and $m_{\mathrm{e}(\mathrm{B})}$ are the electron charge and effective mass in the electrodes (barrier), and the hat^denotes a $2 \times 2$ matrix in spin space.

The eigenstates of the full system $\hat{H}$ are obtained by standard wave matching procedure and the resulting spindependent wave functions $\hat{\psi}_{\mathbf{k}}(x)$ are expressed in terms of a combination of Airy (barrier) and Bessel (ferromagnets) functions. In this picture, the charge and spin currents are defined as

$$
\begin{gathered}
\mathbf{J}_{c}=-\frac{e \hbar}{m} \Im \sum_{i=\mathrm{L}, \mathrm{R}} \int \frac{d^{3} \mathbf{k}}{(2 \pi)^{3}} \hat{\psi}_{\mathbf{k}}^{\dagger}(z) \hat{\nabla} \hat{\psi}_{\mathbf{k}}(z) f_{i}(\mathbf{k}), \\
\mathcal{J}(z)=\frac{\hbar^{2}}{2 m} \mathfrak{s} \sum_{i=\mathrm{L}, \mathrm{R}} \int \frac{d^{3} \mathbf{k}}{(2 \pi)^{3}} \hat{\psi}_{\mathbf{k}}^{\dagger}(z)[\hat{\boldsymbol{\sigma}} \otimes \hat{\nabla}] \hat{\psi}_{\mathbf{k}}(z) f_{i}(\mathbf{k}),
\end{gathered}
$$

with $\mathfrak{\Im}$ referring to the imaginary part and $f_{i}(\mathbf{k})$ being the Fermi-Dirac distribution function of the $i$ th electrode. The spin transfer torque is defined as [6] $\mathbf{T}=-\int_{\mathcal{V}} \nabla \cdot \mathcal{J} d \mathcal{V}$, where $\mathcal{V}$ is the volume of the ferromagnet. In the case of semi-infinite electrodes, the torque exerted on $\mathbf{M}_{R}$ reduces to the interfacial tunneling spin current: $\mathbf{T}=T_{\|} \mathbf{M}_{\mathrm{R}} \times\left(\mathbf{e}_{z} \times\right.$ $\left.\mathbf{M}_{\mathrm{R}}\right)+T_{\perp} \mathbf{M}_{\mathrm{R}} \times \mathbf{e}_{z}$, where $T_{\|}=\mathcal{J}_{\text {int }}^{x}$ and $T_{\perp}=\mathcal{J}_{\text {int }}^{y}$. For the numerical simulations, we choose the parameters describing $\mathrm{Fe} / \mathrm{BaTiO}_{3} / \mathrm{Fe}$ symmetric MTJs (at low temperatures): The effective Fermi energy is $E_{\mathrm{F}}=2.62 \mathrm{eV}$ with an exchange splitting $\Delta=3.86 \mathrm{eV}$ corresponding to the majority (minority) Fermi wave vector $k_{\mathrm{F}}^{\uparrow}=1.09 \AA^{-1}\left(k_{\mathrm{F}}^{\downarrow}=0.4 \AA^{-1}\right)$ and $J=1.07 \mathrm{eV}$. The barrier dielectric permittivity is $\epsilon_{\mathrm{f}}=90 \epsilon_{0}$ and its thickness is $d=1 \mathrm{~nm}$. The barrier height and electron 
effective mass are set to $U_{\mathrm{B}}=2 \mathrm{eV}$ and $m_{\mathrm{B}} / m_{\mathrm{e}}=0.18$. These parameters are extracted from $a b$ initio calculations and correspond to an effective decay wave vector of $q \approx 3 \mathrm{~nm}^{-1}$ (see Ref. [33]). Using these parameters, the screening lengths are $\lambda_{\mathrm{L}(\mathrm{R})}=0.8 \AA$. The typical ferroelectric polarizations (FEP) highlighted in the literature range from 10 to $75 \mu \mathrm{C} / \mathrm{cm}^{2}$ [31,41] and strongly depend on the ferroelectric layer thickness and interfacial structure $[32,33,41]$. Finally, in the present paper, we only focus on the case of a symmetric tunnel junction composed of similar ferromagnetic electrodes.

The interplay between tunnel electroresistance and spin transport has been predicted theoretically $[21,33,38]$ and demonstrated experimentally [23,31,37], allowing for the ferroelectric control of the magnetoresistance and the magnetic control of the electroresistance. The influence of the ferroelectric polarization on the bias dependence of the tunneling magnetoresistance has been addressed within the WKB approximation [42] and from first principles [39]. In the remainder of this paper, we define the TMR and TER as

$$
\begin{aligned}
\mathrm{TMR}_{\leftrightarrow} & =\frac{\mathrm{J}_{\leftrightarrow}^{\mathrm{P}}-\mathrm{J}_{\leftrightarrow}^{\mathrm{AP}}}{\mathrm{J}_{\leftrightarrow}^{\mathrm{AP}}}, \\
\mathrm{TER}^{\mathrm{P}(\mathrm{AP})} & =\frac{\mathrm{J}_{\rightarrow}^{\mathrm{P}(\mathrm{AP})}-\mathrm{J}_{\leftarrow}^{\mathrm{P}(\mathrm{AP})}}{\mathrm{J}_{\leftarrow}^{\mathrm{P}(\mathrm{AP})}} .
\end{aligned}
$$

Here, $\mathrm{J}_{\leftrightarrow}^{\mathrm{P}(\mathrm{AP})}$ denotes the tunneling charge current in parallel (P) and antiparallel (AP) configurations, and $\leftrightarrow$ refers to the leftward (negative) or rightward (positive) ferroelectric polarization of the barrier. The bias dependence of the tunnel magneto- and electroresistance are shown in Figs. 2(a) and 2(b), respectively. The TER and TMR have been calculated for two thicknesses of the tunnel barrier, $d=1 \mathrm{~nm}$ and $1.5 \mathrm{~nm}$, and three values of the FEP, $P=0, \pm 60 \mu \mathrm{C} / \mathrm{cm}^{2}$. In the absence of polarization, the TMR is symmetric as expected in the case of a symmetric MTJ [14,43] [Fig. 2 (a), black symbols]. We note that the bias-induced TMR reversal occurs at large bias voltages $\left(V_{a}>1 \mathrm{~V}\right)$. In the presence of ferroelectric polarization in the barrier, the potential profile becomes asymmetric and the bias dependence of the TMR is heavily distorted, resulting in TMR sign reversal at small voltages. This effect is symmetric when inverting the bias voltage or the FEP. TER has been predicted to emerge in

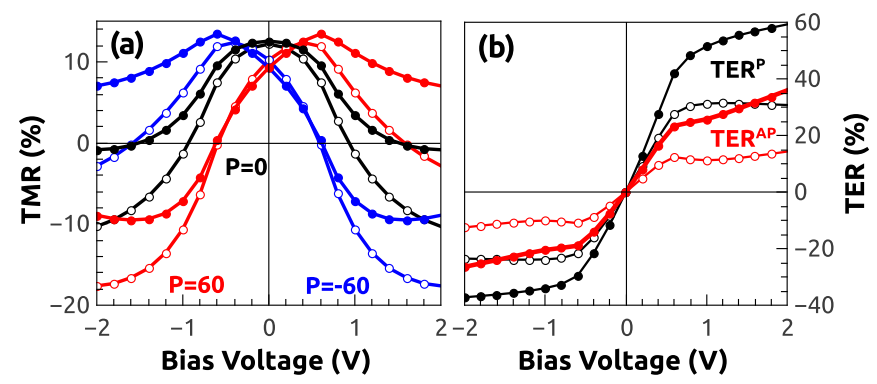

FIG. 2. (Color online) Voltage dependence of (a) TMR for zero (black symbols), positive (red symbols), and negative (blue symbols) FEP and (b) TER for P (black symbols) and AP (red symbols) configurations. The parameters are the same as in the text, with $U_{\mathrm{B}}=2 \mathrm{eV}, P=0, \pm 60 \mu \mathrm{C} / \mathrm{cm}^{2}$, and $d=1 \mathrm{~nm}$ (open symbols) or $1.5 \mathrm{~nm}$ (filled symbols), corresponding to $V_{c}^{\mathrm{L}, \mathrm{R}} \approx 0.35 \mathrm{~V}$ and 0.5 $\mathrm{V}$, respectively.

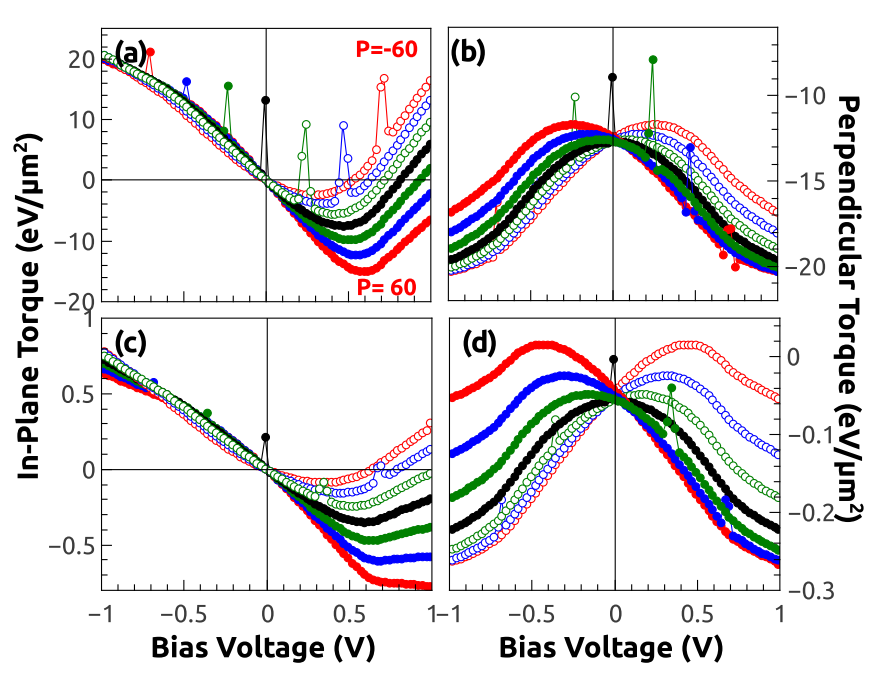

FIG. 3. (Color online) Voltage dependence of the (a,c) in-plane and (b,d) out-of-plane torques exerted on the right layer for $P=$ $0 \mu \mathrm{C} / \mathrm{cm}^{2}$ (black symbols), $P= \pm 20 \mu \mathrm{C} / \mathrm{cm}^{2}$ (green symbols), $P=$ $\pm 40 \mu \mathrm{C} / \mathrm{cm}^{2}$ (blue symbols), and $P= \pm 60 \mu \mathrm{C} / \mathrm{cm}^{2}$ (red symbols). The parameters are $U_{\mathrm{B}}=2 \mathrm{eV}(\mathrm{a}, \mathrm{b}) d=1 \mathrm{~nm}$ and (c,d) $d=1.5 \mathrm{~nm}$, with $\theta=\pi / 2$. The filled and open symbols refer to positive and negative FEP, respectively.

asymmetric tunnel junctions and vanishes in the absence of asymmetry. As shown in Fig. 2(b), the symmetry breaking induced by the bias voltage results in a large TER, even though the MTJ is structurally symmetric. Notice that the TER evaluated for this symmetric MTJ is rather small: TER $\approx 60 \%(40 \%)$ for the $\mathrm{P}(\mathrm{AP})$ magnetic configuration at $V_{a}=2 \mathrm{~V}$. Switching the magnetization configuration results in a strong asymmetric alteration of the bias dependence of the electroresistance effect.

Let us now consider the spin transfer torque exerted on the right layer. Figure 3 displays the bias dependence of the $(\mathrm{a}, \mathrm{c})$ in-plane $T_{\|}\left(V_{a}\right)$ and (b,d) out-of-plane $T_{\perp}\left(V_{a}\right)$ components, respectively for (a,b) $d=1 \mathrm{~nm}$ and for (c,d) $d=1.5 \mathrm{~nm}$, with $U_{B}=2 \mathrm{eV}$. Each curve displays a noisy peak when the barrier shape becomes rectangular due to computational inaccuracy in the treatment of Airy functions. This peak is broader when the barrier is thinner $(d=1 \mathrm{~nm})$. One directly sees that tuning the ferroelectric polarization of the barrier modifies the bias dependence of both in-plane and out-of-plane torque components. This modulation resembles the one observed when using structurally asymmetric tunnel junctions $[14,44]$ : the zero-bias slope of the in-plane torque, $\partial T_{\|} / \partial V$, increases (decreases) for a positive (negative) FEP, while the perpendicular torque $T_{\perp}$ acquires a large positive (negative) linear bias component for a negative (positive) FEP. These features demonstrate that an efficient control over the STT magnitude can be achieved using ferroelectricity.

In Fig. 4(a) the critical voltage at which the in-plane torque $T_{\|}$changes sign is represented as a function of the barrier thickness for different values of the ferroelectric polarization, $P=0, \pm 40, \pm 60 \mu \mathrm{C} / \mathrm{cm}^{2}$. For zero and positive FEP, the critical voltage increases with the barrier thickness and diverges around $d=1.2-1.5 \mathrm{~nm}$. Beyond this point, the in-plane torque does not change sign at all as a function of 


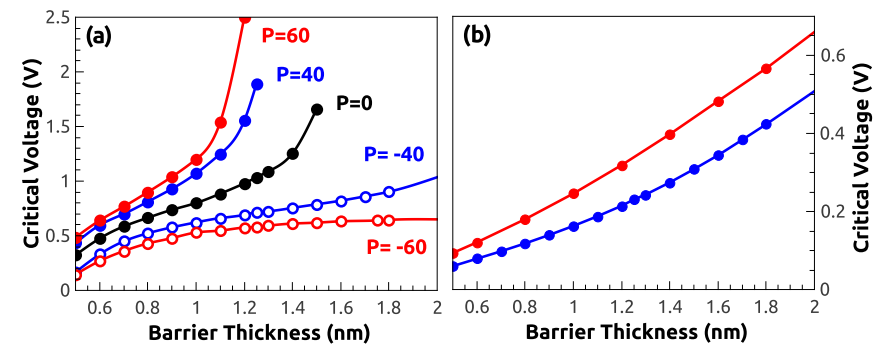

FIG. 4. (Color online) (a) Dependence of the critical voltage at which the in-plane torque changes sign as a function of the barrier thickness for $P \in[-60,+60] \mu \mathrm{C} / \mathrm{cm}^{2} ;$ (b) dependence of the critical voltage at which the out-of-plane torque reaches its extremum as a function of the barrier thickness for $P=40$ and $60 \mu \mathrm{C} / \mathrm{cm}^{2}$. The barrier height is $U_{\mathrm{B}}=2 \mathrm{eV}$.

the bias voltage. In contrast, for negative FEP, the critical voltage seems to saturate around $0.5 \mathrm{~V}$ for the set of parameters adopted in the simulations. It therefore clearly appears that the critical voltage at which the in-plane torque changes sign can be strongly modified by reverting the direction of the FEP. This provides an interesting tool for extremely efficient control of the torque in magnetic tunnel junctions: By switching the FEP direction, it is possible to obtain an in-plane torque with an almost quadratic bias dependence (for negative FEP) or a mostly linear bias dependence (for positive FEP). Notice that the specific values provided in Fig. 4(a) depend on the height and thickness of the barrier and on the effective mass of the tunneling electron. However, the tendencies displayed in Figs. 3 and 4 are quite general. The bias dependence of the out-of-plane torque itself is very similar to what is observed in structurally or impurity wise asymmetric MTJs [14,44]. The out-of-plane torque acquires a component with a linear bias dependence, which shifts the critical voltage at which the out-of-plane torque reaches its extremum [see Figs. 3(b) and 3(d)]. This critical voltage increases almost linearly with the barrier thickness, as displayed in Fig. 4(b).

In order to elucidate the role of the interfacial charge depletion/accumulation regions on the spin torque, we also calculated the spin transfer torque in the absence of these regions, while keeping the asymmetry in the barrier potential. To do so, we simply replace $\Delta_{\mathrm{L}(\mathrm{R})}(z)$ and $e V(z)$ [see Eqs. (3) and (4)] by $\Delta$ and $e V_{a}$, respectively. Hence, the situation is the very same as regular MTJs with structural asymmetry as studied in Refs. [14,44]. The additional barrier potential due to the ferroelectricity at the left and right interfaces for $P= \pm 40 \mu \mathrm{C} / \mathrm{cm}^{2}$ are $V_{c}^{\mathrm{L}, \mathrm{R}} \approx \pm 0.23 \mathrm{~V}$ for $d=1 \mathrm{~nm}$ and $V_{c}^{\mathrm{L}, \mathrm{R}} \approx \pm 0.34 \mathrm{~V}$ for $d=1.5 \mathrm{~nm}$.

The resulting modulation of the torque being quite small (not shown), we choose to display the electrical efficiency of the in-plane torque $T_{\|} / j_{e}$ to illustrate this effect. The results are displayed in Figs. 5(a) and 5(b) for $d=1 \mathrm{~nm}$ and $1.5 \mathrm{~nm}$, respectively. The open symbols represent the efficiency in the absence of ferroelectricity (i.e. for the equivalent structurally asymmetric barrier as explained above), while the filled symbols represent the efficiency of the torque in the ferroelectric junction. First, we notice that the modulation of the torque efficiency by tuning the FEP can be quite large (see filled symbols) and increases with the barrier

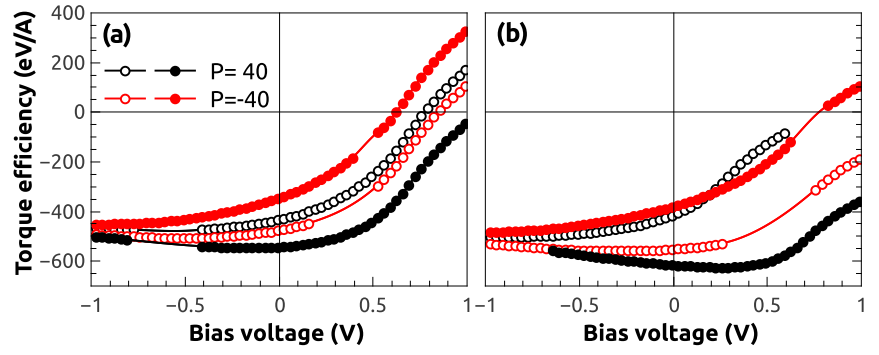

FIG. 5. (Color online) Bias dependence of the efficiency of the in-plane torque, $T_{\|} / j_{e}$, for a barrier thickness (a) $d=1 \mathrm{~nm}$ and (b) $d=1.5 \mathrm{~nm}$. The filled symbols represent the efficiency calculated with $P= \pm 40 \mu \mathrm{C} / \mathrm{cm}^{2}$ (black and red symbols, respectively), corresponding to (a) $V_{c}^{\mathrm{L}, \mathrm{R}} \approx 0.23 \mathrm{~V}$ and (b) $0.34 \mathrm{~V}$, as explained in the text. The open symbols represent the efficiency calculated with a normal insulating barrier with asymmetric potential, i.e. in the absence of the interfacial depletion/accumulation region. The average barrier height is $U_{\mathrm{B}}=2 \mathrm{eV}$ in all the cases.

thickness. Second, the modulation of the torque efficiency induced by structural asymmetry (open symbols) is overall weaker than the one induced by FEP. Furthermore, the sign of the modulation due to a ferroelectric barrier is actually opposite to the one obtained using an asymmetric normal tunnel barrier. In the case of positive structural asymmetry $\left(V_{c}^{\mathrm{L}}-V_{c}^{\mathrm{R}}>0\right.$, black open symbols), the efficiency is reduced, while it is increased in the case of positive FEP (black filled symbols). This points out the importance of interfacial charge depletion/accumulation region. Although these effects occur on very short distances (less than $1 \AA$ ), they dramatically affect the spin-dependent tunneling, since tunneling is mainly controlled by the interfacial densities of states.

To complete our study, we plot in Fig. 6 the spatial dependence of the transverse spin density (a,b,c) $S_{x}$ and $(\mathrm{d}, \mathrm{e}, \mathrm{f}) S_{y}$ in the right layer for different FEP $(P=-40$,

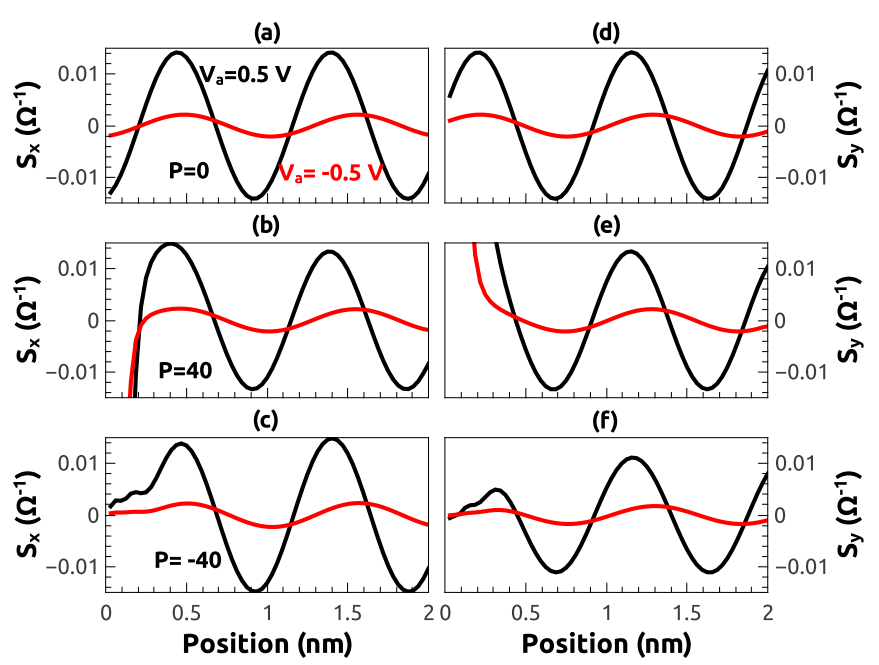

FIG. 6. (Color online) Spatial dependence of the spin density (a,b,c) $S_{x}$ and (d,e,f) $S_{y}$ in the right electrode calculated at the Fermi energy and perpendicular incidence $k=0$. The parameters are (a,d) $P=0 \mu \mathrm{C} / \mathrm{cm}^{2}$, (b,e) $P=40 \mu \mathrm{C} / \mathrm{cm}^{2}$ and (c,f) $P=-40 \mu \mathrm{C} / \mathrm{cm}^{2}$ and $V_{a}= \pm 0.5 \mathrm{~V}$ (black and red, respectively). The units are given in inverse volume of the unit cell $\Omega^{-1}$. 
0 , and $\left.+40 \mu \mathrm{C} / \mathrm{cm}^{2}\right)$ and bias voltage $\left(V_{a}= \pm 0.5 \mathrm{~V}\right)$. This spin accumulation is calculated for Fermi electrons with a normal incidence, for simplicity and tractability. In the absence of ferroelectric polarization, the spatial dependence of the spin density oscillates regularly, as expected in regular tunnel junctions [13] [see Figs. 6(a) and 6(d)]. However, when the FEP is positive [see Figs. 6(b) and 6(e)], the spin density increases dramatically in the depletion region, which corresponds to an enhancement of the torque efficiency (see Fig. 5). Conversely, when the FEP is negative [see Figs. 6(c) and 6(f)], the spin density is strongly reduced in the accumulation region, which corresponds to a reduction of the torque efficiency (see Fig. 5). Considering that the spin transfer torque results from the spatial averaging of the spin density over the volume of the ferromagnet, only the spin density close to the interface significantly contributes to the torque (the remaining spin density averages out under dephasing far in the ferromagnetic electrode [13]). Therefore, the presence of a depletion or accumulation region close to the interface has a substantial impact on the bias dependence of the torque. This contribution is actually much larger than the one coming from the FEP-induced barrier structural asymmetry [14] or impurity-induced asymmetry [44].

The ferroelectric control of the bias dependence of both in-plane and out-of-plane components of the spin torque has important consequences in terms of current-driven magnetization switching and dynamics. It is usually accepted that in-plane torque drives the magnetization switching and self-sustained precessions [2,3]. Therefore, a smart design of the ferroelectric junction enables the efficient control of the dynamical properties by tuning both the injected current and ferroelectric polarization. Another interesting aspect is the control of the out-of-plane torque. In a recent publication, Oh et al. [17] demonstrated the important role of the out-of-plane component on current-driven magnetic instabilities (tagged switching back phenomenon) in MTJs. In the light of the present study, controlling this effect through ferroelectricity seems a viable route to reduce these instabilities which in particular may be a crucial nuisance for STT-MRAM. Since the ferroelectric polarization is usually controlled through voltage pulses, spin torque and FEP switching can be combined to obtain original device operation modes.

Finally, two important aspects are to be considered regarding the applicability of the present theory to experimental situations. First, the model we use in the present paper is a two band Stoner model applied to a symmetric junction. It disregards the particularities of the realistic band structure of the electrodes and of the barrier as well as possible wave function filtering effects or resonant states. However, it possesses the minimal ingredients of ferroelectricity coexisting with spin splitting, which should capture most of the relevant physics at stake. Within this model, the TER obtained numerically is small compared to the experimental observations: about $40 \%$ to $60 \%$ in the present work compared to $10000 \%$ [37] and $75000 \%$ [23] in thick $\mathrm{BaTiO}_{3}$ with asymmetric magnetic electrodes. This indicates that the modulation of the torque efficiency expected experimentally in asymmetric ferroelectric magnetic tunnel junctions should actually be much larger than the one derived in the present paper. Consequently, one can expect that the ferroelectric control of the spin torque efficiency in realistic asymmetric MTJs should be much more efficient than the results reported here. Second, whereas the voltage control of tunneling electroresistance has been recently realized in thick $\mathrm{BaTiO}_{3}$-based MTJs [23,37], the realization of spin transfer torque in thin $\mathrm{BaTiO}_{3}$ junctions presents the advantage of generating unique current-driven dynamical states that are absent in thick ferroelectric junctions. The combination of ferroelectricity with spin transfer torque is thus expected to produce a wealth of original states of major interest for technologists and researchers.

We thank M. Zhuravlev and N. Useinov for useful conversations and support, and E.Y. Tsymbal for his critical review of the manuscript and his constructive comments. This work was supported by the King Abdullah University of Science and Technology (KAUST).
[1] C. Chappert, A. Fert, and F. Nguyen Van Dau, Nat. Mater. 6, 813 (2007); J. A. Katine and E. E. Fullerton, J. Magn. Magn. Mater. 320, 1217 (2008).

[2] A. Brataas, A. D. Kent, and H. Ohno, Nat. Mater. 11, 372 (2012).

[3] D. Ralph and M. Stiles, J. Magn. Magn. Mater. 320, 1190 (2008).

[4] M. Tsoi, in Handbook of Spin Transport and Magnetism, edited by E.-Y. Tsymbal and I. Zutic, Chap. 7 (CRC Press, Boca Raton, 2011); A. Manchon, and S. Zhang, in Handbook of Spin Transport and Magnetism, edited by E.-Y. Tsymbal and I. Zutic, Chap. 8 (CRC Press, Boca Raton, 2011).

[5] T. Maruyama, Y. Shiota, T. Nozaki, K. Ohta, N. Toda, M. Mizuguchi, A. A. Tulapurkar, T. Shinjo, M. Shiraishi, S. Mizukami, Y. Ando, and Y. Suzuki, Nat. Nanotechnol. 4, 158 (2009); Y. Shiota, T. Nozaki, F. Bonell, S. Murakami, T. Shinjo, and Y. Suzuki, Nat. Mater. 11, 39 (2012).

[6] J. C. Slonczewski, J. Magn. Magn. Mater. 159, L1 (1996); L. Berger, Phys. Rev. B 54, 9353 (1996).
[7] D. Houssameddine, U. Ebels, B. Delaet, B. Rodmacq, I. Firastrau, F. Ponthenier, M. Brunet, C. Thirion, J.-P. Michel, L. Prejbeanu-Buda, M.-C. Cyrille, O. Redon, and B. Dieny, Nat. Mater. 6, 447 (2007).

[8] M. Hosomi, H. Yamagishi, T. Yamamoto, K. Bessho, Y. Higo, K. Yamane, H. Yamada, M. Shoji, H. Hachino, C. Fukumoto, H. Nagao, and H. Kano, IEDM Techn. Digest, 459 (2005); T. Kawahara, R. Takemura, K. Miura, J. Hayakawa, S. Ikeda, Y. M. Lee, R. Sasaki, Y. Goto, K. Ito, I. Meguro, F. Matsukura, H. Takahashi, H. Matsuoka, and H. Ohno, IEEE J. Solid State Circ., 43, 109 (2008).

[9] S. S. P. Parkin, M. Hayashi, and L. Thomas, Science 320, 190 (2008).

[10] S. S. P. Parkin, C. Kaiser, A. Panchula, P. M. Rice, B. Hughes, M. Samant, and S.-H. Yang, Nat. Mater. 3, 862 (2004); S. Yuasa, T. Nagahama, A. Fukushima, Y. Suzuki, and K. Ando, ibid. 3, 868 (2004). 
[11] F. Bonell, T. Hauet, S. Andrieu, F. Bertran, P. Le Fevre, L. Calmels, A. Tejeda, F. Montaigne, B. Warot-Fonrose, B. Belhadji, A. Nicolaou, and A. Taleb-Ibrahimi, Phys. Rev. Lett. 108, 176602 (2012).

[12] See, e.g., J. P. Velev, P. A. Dowben, E. Y. Tsymbal, S. J. Jenkins, and A. N. Caruso, Surf. Sci. Rep. 63, 400 (2008).

[13] I. Theodonis, N. Kioussis, A. Kalitsov, M. Chshiev, and W. Butler, Phys. Rev. Lett. 97, 237205 (2006); C. Heiliger and M. D. Stiles, ibid. 100, 186805 (2008); J. Xiao, G. E. W. Bauer, and A. Brataas, Phys. Rev. B 77, 224419 (2008); A. Manchon, N. Ryzhanova, A. Vedyayev, M. Chschiev, and B. Deny, J. Phys.: Condens. Matter 20, 145208 (2008).

[14] M. Wilczynski, J. Barnas, and R. Swirkowicz, Phys. Rev. B 77, 054434 (2008); Y.-H. Tang, N. Kioussis, A. Kalitsov, W. H. Butler, and R. Car, Phys. Rev. Lett. 103, 057206 (2009); Phys. Rev. B 81, 054437 (2010); A. Manchon, S. Zhang, and K.-J. Lee, ibid. 82, 174420 (2010).

[15] Y. Huai, F. Albert, P. Nguyen, M. Pakala, and T. Valet, Appl. Phys. Lett. 84, 3118 (2004); G. D. Fuchs, N. C. Emley, I. N. Krivorotov, P. M. Braganca, E. M. Ryan, S. I. Kiselev, J. C. Sankey, D. C. Ralph, R. A. Buhrman, and J. A. Katine, ibid. 85, 1205 (2004).

[16] J. C. Sankey, Y.-T. Cui, J. Z. Sun, J. C. Slonczewski, R. A. Buhrman, and D. C. Ralph, Nat. Phys. 4, 67 (2008); H. Kubota, A. Fukushima, K. Yakushiji, T. Nagahama, S. Yuasa, K. Ando, H. Maehara, Y. Nagamine, K. Tsunekawa, D. D. Djayaprawira, N. Watanabe, and Y. Suzuki, 4, 37 (2008).

[17] S.-C. Oh, S.-Y. Park, A. Manchon, M. Chshiev, J.-H. Han, H.-W. Lee, J.-E. Lee, K.-T. Nam, Y. Jo, Y.-C. Kong, B. Dieny, and K.-J. Lee, Nat. Phys. 5, 898 (2009).

[18] A. Manchon, R. Matsumoto, H. Jaffres, and J. Grollier, Phys. Rev B 86, 060404(R) (2012).

[19] W. Eerenstein, N. D. Mathur, and J. F. Scott, Nature (London) 442, 759 (2006); H. Bea, M. Gajek, M. Bibes, and A. Barthélémy, J. Phys.: Condens. Matter 20, 434221 (2008).

[20] L. Esaki, R. B. Laibowitz, and P. J. Stiles, IBM Tech. Discl. Bull. 13, 2161 (1971).

[21] M. Ye. Zhuravlev, R. F. Sabirianov, S. S. Jaswal, and E. Y. Tsymbal, Phys. Rev. Lett. 94, 246802 (2005); M. Ye. Zhuravlev, S. S. Jaswal, E. Y. Tsymbal, and R. F. Sabirianov, Appl. Phys. Lett. 87, 222114 (2005).

[22] D. I. Bilc, F. D. Novaes, J. Iniguez, P. Ordejon, and P. Ghosez, ACS Nano 6, 1473 (2012).

[23] V. Garcia, S. Fusil, K. Bouzehouane, S. Enouz-Vedrenne, N. D. Mathur, A. Barthélémy, and M. Bibes, Nature (London) 460, 81 (2009).

[24] A. Gruverman, D. Wu, H. Lu, Y. Wang, H. W. Jang, C. M. Folkman, M. Ye. Zhuravlev, D. Felker, M. Rzchowski, C.-B. Eom, and E. Y. Tsymbal, Nano Lett. 9, 3539 (2009).

[25] A. Crassous, V. Garcia, K. Bouzehouane, S. Fusil, A. H. G. Vlooswijk, G. Rispens, B. Noheda, M. Bibes, and A. Barthélémy, Appl. Phys. Lett. 96, 042901 (2010).

[26] D. Pantel, H. Lu, S. Goetze, P. Werner, D. J. Kim, A. Gruverman, D. Hesse, and M. Alexe, Appl. Phys. Lett. 100, 232902 (2012).

[27] L. Jiang, W. S. Choi, H. Jeen, S. Dong, Y. Kim, M.-G. Han, Y. Zhu, S. V. Kalinin, E. Dagotto, T. Egami, and H. N. Lee, Nano Lett. 13, 5837 (2013).

[28] H. Yamada, V. Garcia, S. Fusil, S. Boyn, M. Marinova, A. Gloter, S. Xavier, J. Grollier, E. Jacquet, C. Carrétéro, C. Deranlot,
M. Bibes, and A. Barthélémy, ACS Nano 7, 5385 (2013).

[29] Z. Wen, C. Li, D. Wu, A. Li, and N. Ming, Nat. Mater. 12, 617 (2013).

[30] E. Tsymbal and H. Kohlstedt, Science 313, 181 (2006).

[31] V. Garcia, M. Bibes, L. Bocher, S. Valencia, F. Kronast, A. Crassous, X. Moya, S. Enouz-Vedrenne, A. Gloter, D. Imhoff, C. Deranlot, N. D. Mathur, S. Fusil, K. Bouzehouane, and A. Barthélémy, Science 327, 1106 (2010); D. Pantel, S. Goetze, D. Hesse, and M. Alexe, Nat. Mater. 11, 289 (2012).

[32] S. Valencia, A. Crassous, L. Bocher, V. Garcia, X. Moya, R. O. Cherifi, C. Deranlot, K. Bouzehouane, S. Fusil, A. Zobelli, A. Gloter, N. D. Mathur, A. Gaupp, R. Abrudan, F. Radu, A. Barthélémy, and M. Bibes, Nat. Mater. 10, 753 (2011); L. Bocher, A. Gloter, A. Crassous, V. Garcia, K. March, A. Zobelli, S. Valencia, S. Enouz-Vedrenne, X. Moya, N. D. Marthur, C. Deranlot, S. Fusil, K. Bouzehouane, M. Bibes, A. Barthélémy, C. Colliex, and O. Stephan, Nano Lett. 12, 376 (2012).

[33] J. P. Velev, Ch.-G. Duan, J. D. Burton, A. Smogunov, M. K. Niranjan, E. Tosatti, S. S. Jaswal, and E. Y. Tsymbal, Nano Lett. 9, 427 (2009); J. P. Velev, C.-G. Duan, K. D. Belashchenko, S. S. Jaswal, and E. Y. Tsymbal, Phys. Rev. Lett. 98, 137201 (2007); J. D. Burton and E. Y. Tsymbal, ibid. 106, 157203 (2011).

[34] Y. W. Yin, J. D. Burton, Y-M. Kim, A. Y. Borisevich, S. J. Pennycook, S. M. Yang, T. W. Noh, A. Gruverman, X. G. Li, E. Y. Tsymbal, and Q. Li, Nat. Mater. 12, 397 (2013).

[35] R. O. Cherifi, V. Ivanovskaya, L. C. Phillips, A. Zobelli, I. C. Infante, E. Jacquet, V. Garcia, S. Fusil, P. R. Briddon, N. Guiblin, A. Mougin, A. A. Unal, F. Kronast, S. Valencia, B. Dkhil, A. Barthlmy, and M. Bibes, Nat. Mater. 13, 345 (2014).

[36] H. Yamada, M. Marinova, P. Altuntas, A. Crassous, L. BégonLours, S. Fusil, E. Jacquet, V. Garcia, K. Bouzehouane, A. Gloter, J. E. Villegas, A. Barthélémy, and M. Bibes, Sci. Rep. 3, 2834 (2013).

[37] A. Chanthbouala, A. Crassous, V. Garcia, K. Bouzehouane, S. Fusil, X. Moya, J. Allibe, B. Dlubak, J. Grollier, S. Xavier, C. Deranlot, A. Moshar, R. Proksch, N. D. Mathur, M. Bibes, and A. Barthélémy, Nat. Nanotechnol. 7, 101 (2012); A. Chanthbouala, V. Garcia, R. O. Cherifi, K. Bouzehouane, S. Fusil, X. Moya, S. Xavier, H. Yamada, C. Deranlot, N. D. Mathur, M. Bibes, A. Barthélémy, and J. Grollier, Nat. Mater. 11, 860 (2012).

[38] M. Y. Zhuravlev, S. Maekawa, and E. Y. Tsymbal, Phys. Rev. B 81, 104419 (2010).

[39] N. M. Caffrey, T. Archer, I. Rungger, and S. Sanvito, Phys. Rev. B 83, 125409 (2011).

[40] S. Zhang, Phys. Rev. Lett. 83, 640 (1999).

[41] A. Petraru, N. A. Pertsev, H. Kohlstedt, U. Poppe, R. Waser, A. Solbach, and U. Klemradt, J. Appl. Phys. 101, 114106 (2007); L. Pintilie, I. Boerasu, M. J. M. Gomes, T. Zhao, R. Ramesh, and M. Alexe, ibid. 98, 124104 (2005).

[42] N. A. Zimbovskaya, J. Appl. Phys. 106, 124101 (2009).

[43] E. Y. Tsymbal, O. N. Mryasov, and P. R. LeClair, J. Phys.: Condens. Matter 15, R109 (2003).

[44] A. Kalitsov, W. Silvestre, M. Chshiev, and J. P. Velev, Phys. Rev. B 88, 104430 (2013). 